\title{
La Diffusion Des Contenus Televisuels Et Les Pratiques De Reception Des Spectateurs A L'ere Du Numerique
}

\author{
Kahi Oulaï Honoré, (Docteur en communication)
}

Université AO de Bouaké, Côte d'Ivoire

doi: 10.19044/esj.2016.v12n33p198 URL:http://dx.doi.org/10.19044/esj.2016.v12n33p198

\begin{abstract}
The objective is to question broadcasting methods and TV reception practices in the digital area. In this logic, we first adopt a literary review and then an investigation using a questionnaire and a semi-directing talk. The data show that media convergence is conceptualized in its technological and cultural parameters. It is perceived like a change of perspective to figure out the evolution of media and its various uses in terms of broadcasting process and consumption posture. The broadcasting processes concern the exploitation of the multiplicity of screens, methods of publication and the use of sociotechnical devices. The individual and collective practices of consumption vary according to the user's technical and technological skills which induce various viewing postures.
\end{abstract}

Keywords: Convergence, broadcasting, position, practice, screen

\section{Résumé}

L'objectif est d'interroger les modes de diffusion et les pratiques de réception de la télévision à l'ère du numérique. A cette fin, nous optons d'abord pour une revue documentaire et ensuite pour une enquête par questionnaire et des entretiens semi-directifs. Les résultats obtenus montrent que la convergence médiatique est conceptualisée dans ses paramètres technologique et culturel. Elle est perçue comme un changement de vision pour comprendre l'évolution médiatique et ses divers usages en termes de processus de diffusion et de postures de consommation. Les processus de diffusion portent sur l'exploitation de la multiplicité des écrans, les modalités de publication et le recours aux dispositifs sociotechniques. Les pratiques individuelles et les pratiques collectives de consommation varient selon les compétences techniques et technologiques qui induisent des postures spectatorielles diverses. 
Mots-clés : convergence, diffusion, posture, pratique, écran

\section{Introduction}

L'avènement des satellites, des bouquets de chaînes, des offres multimédia (Virginie SONET, 2012) et des nouveaux services associés aux télévisions connectées a profondément modifié l'environnement audio-visuel (Myriam Montagut-Lobjoit, 2010) et par ricochet, les processus de diffusion des contenus télévisuels. En outre, le développement des équipements numériques et de tout l'écosystème que constituent les terminaux - baladeurs vidéo, tablettes, smartphones, ordinateurs etc. - (CNC, 2012) a entraîné une évolution des pratiques audiovisuelles en régime numérique et une reconfiguration des rapports entre acteurs historiques et les nouveaux acteurs, ceux du web. Les sites des émissions, les blogs, les réseaux sociaux numériques, s'ajoutent aux multiples écrans de visionnage pour modifier les logiques de programmation télévisuelles (Perticoz et Dessinges, 2015).

Ces objets techniques et applications logicielles participent à la diversification des accès aux contenus télévisés et à la mutation des pratiques et usages des spectateurs. Ainsi, selon Elodie K. et Florence R. (2015), la télévision, en intégrant les nouvelles technologies numériques, transforme son rapport aux programmes, aux supports audiovisuels et aux spectateurs. Ces mutations invitent à repenser la pluralité des formes et le réajustement des modes de consommation des contenus et des circonstances de visionnement (Elodie K. et Florence R.). Perticoz et Dessinges (2015), par le biais d'une modélisation suggèrent que la figure traditionnelle du téléspectateur soit remplacée par celle du télévisionneur. Ils spécifient les nouvelles postures à l'œuvre (Perticoz et Dessinges, 2015) ainsi que les trois axes structurants des pratiques de consommation. Leur outil conceptuel permet de cerner les modes de visionnage et de favoriser la compréhension des mutations. Ces mutations induisent un environnement soumis à une logique de délinéarisation (Lacombes, 2015) des contenus télévisuels.

Les pratiques de visionnage en résultant ont été reparties en deux grands modes de consommation (Idem). L'un des modes s'inscrit dans le principe du rendez-vous télévisuel récurrent et l'autre est caractérisé par la prise en main de la temporalité spectatorielle par le spectateur. Lacombes en fait une description détaillée explicitant chaque mode de visionnage. Dans le même souffle, Guillaume Blanc (2015), Amandine K. et Marlène L. (2015) mènent respectivement des études sur les pratiques de réception télévisuelles dans les foyers et chez les publics jeunes. Le premier montre que les possibilités techniques offertes par le numérique participent à une réappropriation de l'espace temps-foyer et à la complémentarité des usages et des cadres de réception (Guillaume Blanc, 2015). Les seconds montrent que 
les pratiques des publics jeunes laissent entrevoir une articulation entre permanences et mutations (Amandine K. et Marlène L., 2015).

Au total, ces études présentent un caractère convergent : la manière dont Internet et les outils numériques redessinent les contours de l'objet télévisuel, de la production à la réception et des pratiques aux usages. C'est surtout ce caractère homogène des travaux consultés qui justifie un regard unifié des études sur les pratiques émergentes dans le paysage audiovisuel. Dans cette optique, cet article interroge d'abord les modes de diffusion des contenus télévisuels et ensuite les pratiques de réception des usagers. En d'autres termes, il s'agira pour nous de questionner les tendances qui se dessinent et de reconsidérer les nouveaux enjeux d'un dispositif médiatique en perpétuel reconstruction. Pour pouvoir répondre à cette question, nous partons de la théorie de la « convergence médiatique » (Henry Jenkins, 2006 et 2013 ; Elizabeth Bastos Duarte et al.; 2011) pour examiner les mutations en rapport avec les processus de diffusion des contenus télévisuels. Le modèle du sentiment d'efficacité personnelle face aux technologies, développé par Compeau et Higgins (1995), sera utilisé pour comprendre la diversification des pratiques de consommation. Par ailleurs, notre lecture du contexte actuel de la télévision sous le regard croisé des deux théories induit l'hypothèse selon laquelle les processus de diffusion réfèrent à l'agrégation et à la programmation ; les pratiques de réception quant à elles, renvoient aux logiques d'usage que sont : l'appropriation, la co-construction et l'autonomie.

La méthodologie utilisée pour vérifier cette hypothèse a consisté en des recherches documentaires et à l'application des méthodes d'enquête mixte combinant les approches quantitative et qualitative. La recherche documentaire a concerné les bibliothèques de l'Université de Bouaké principalement et secondairement notre fonds documentaire personnel. Cette recherche a permis de faire l'état des lieux de la question à l'étude et de renforcer nos connaissances à partir des livres et articles publiés. Pour les entretiens, des personnes physiques (étudiants, fans des séries télévisés, gestionnaire des télécentres et foyers) ont été approchées en vue d'avoir des informations sur leurs pratiques de visionnement. Enfin pour l'observation directe, nous nous sommes rendus dans des télécentres publics (ici appelés cybercafés), sur le campus et dans les domiciles pour observer respectivement les fans, les étudiants et les foyers dans leurs postures spectatorielles. Les données collectées ont été analysées pour faire ressortir les tendances essentielles à l'étude et organiser la réflexion sur trois axes : l'aperçu des logiques de la convergence médiatique, les processus de diffusion et la diversification des pratiques de consommation.

\section{Aperçu des logiques de la convergence médiatique}

Le déploiement des plates-formes médiatiques a permis la mise en 
place de nouvelles stratégies de production dans les épisodes par les producteurs de séries télévisées. Cela constitue une preuve de l'entrée dans l'ère de la « techno-télévision » (Mélanie Bourdaa, 2011) dont la caractéristique principale est la convergence médiatique (Jenkins, 2013). Selon Duarte et al. (2011), la convergence médiatique implique le recours à des plates-formes différentes, à savoir un ensemble de dispositifs et de supports technologiques pour réaliser, véhiculer et consommer les programmes télévisuels. Cette convergence articule des formats, des langages et des esthétiques sur divers écrans, ouvrant des possibilités pour de nouveaux modes d'interaction avec des récepteurs qui, peu à peu, sont en train d'abandonner leur condition de simples spectateurs pour devenir des utilisateurs et des producteurs.

François JOST (2015) souligne que la possibilité existe pour les produits télévisuels de recourir à des technologies d'autres médias, tant dans leurs processus de réalisation que par la diffusion sur d'autres plates-formes. Ces produits peuvent convoquer ces autres plates-formes soit par insertion dans la trame narrative elle-même soit par interaction avec le spectateur ; ils peuvent donner une extension à leurs récits sur d'autres plates-formes ou selon l'expression en usage, « extensions transmédiatiques interactives » (Mélanie Bourdaa, 2011).

Jenkins rattache l'émergence des récits transmédias au fait que différents médias coexistent sans s'annuler et entrent de plus en plus facilement en résonnance. Les smartphones qui permettent à la fois de surfer sur le web, de voir des films, d'écouter de la musique, de jouer ou de prendre des photos en sont la matérialisation. Jenkins lui-même donne de la convergence, la définition suivante : «Par convergence j'entends le flux de contenu passant par de multiples plates-formes médiatiques, la coopération entre une multitude d'industries médiatiques et le comportement migrateur des publics et des médias qui, dans leur quête d'expériences et de divertissement qui leur plaisent, vont et fouillent partout. ». (Jenkins, 2013). Il ressort des travaux de Jenkins, deux dimensions de la convergence médiatique : la dimension technologique et la dimension culturelle.

Par ailleurs, le paradigme de la convergence médiatique, aux dires de Jenkins, repose sur l'hypothèse selon laquelle les médias anciens vont interagir avec les médias nouveaux de la façon la plus complexe (Hubert Guillaud, 2013). Frédéric Martel (2013) souligne que cette complexité n'est pas aisée à cerner du fait des innombrables formes médiatiques sans cesse réinventées. Les médias ont dû coexister avec les médias nouveaux (Lisa Gitelman, 2008), ce qui rend plus crédible le paradigme de la convergence médiatique au-delà du fait qu'il soit corrélé à la théorie du même nom. « (...) la théorie de la convergence médiatique implique deux critères décisifs : l'expérience culturelle de masse, et l'appropriation des contenus à travers 
des machines, des appareils, bref des médias, électriques d'abord, numériques, ensuite. " (Olivier Aïn, 2015). Ces deux critères réfèrent respectivement à la logique technologique et à la logique culturelle de la convergence médiatique.

La logique technologique de la convergence renvoie aux :

- Mouvements de condensation ou d'expansion des récits (François JOST, 2011) avec leurs deux variantes, l'inhérence et l'adhérence. L'inhérence est l'intériorisation de l'articulation entre le produit et la ou les plates-formes dans les limites du texte télévisuel ; l'adhérence en est le contraire en plus de son déploiement dans d'autres médias.

- principe de sérialité qui consiste à capter et garder l'attention du téléspectateur et l'engager dans la réception par le modèle d'épisode. En plus de faire revenir le téléspectateur chaque semaine devant son écran, les producteurs mettent en place des "previously on" ou résumés courts des épisodes précédents qui permettent de s'approprier les arcs narratifs. Une autre stratégie d'accroche ou "cliffhanger" (scène choc) de fin d'épisode va créer une attente de la suite (Mélanie Bourdaa, 2011).

- $\quad$ extensions transmédiatiques qui permettent de créer un univers sériel complexe, immersif et interactif en tirant partie de toutes les plates-formes médiatiques disponibles (jeux vidéo, Internet, réseaux sociaux numériques, films...). Les créations et extensions transmédiatiques enrichissent les univers sériels et favorisent l'engagement et l'immersion des fans.

La logique culturelle de la convergence réfère :

- $\quad$ à ce que recouvre la convergence pour les consommateurs comme un processus qui implique la formation et le développement de communautés participatives. En même temps que les consommateurs sont invités à butiner d'un média à l'autre, ils peuvent aussi de plus en plus facilement intervenir sur les maillons de la chaîne, se les approprier, les échanger, les commenter, les transformer et les diffuser.

- aux activités des simples usagers : ce sont les activités de visionnement qui respectent la sérialité programmée dans la logique de la télévision hertzienne.

- aux activités des fans : ce sont des activités créatives et collaboratives. Mélanie Bourdaa (2011) les catégorise en quatre : les activités de création, les créations de lien social, les créations de partage et les créations d'intelligence collective.

Pour Duarte et al. (2011), tout le mouvement de convergence cherche à correspondre au profil du consommateur contemporain, perçu dans ses interactions sociales, dans ses façons de consommer et ses relations avec les technologies du moment pour cerner, outrepassant de beaucoup les aspects techniques pour acquérir une dimension culturelle et économique (Jost 
François, 2011). Duarte et al. soulignent que la convergence est un processus évolutif qui intègre non seulement différentes technologies, mais qui est attentif aussi à l'apparition de nouvelles options. Somme toute, nous retiendrons que la convergence médiatique, conceptualisée par les travaux cités dans ses paramètres non seulement technologiques mais surtout culturels, est considérée comme un changement de paradigme pour comprendre l'évolution médiatique et ses usages divers. Dans les deux sections qui suivent, nous aborderons les axes suivants : les processus de diffusion et la diversification des pratiques de consommation.

\section{Les processus de diffusion}

Nous rappelons que pour mieux comprendre ces processus, nous utilisons ici une méthodologie qui repose sur l'étude documentaire. Les variables retenues sont en rapport avec le choix des objets techniques, la place croissante de l'Internet dans l'accès aux contenus télévisés, les préférences médiatiques, les écrans multiples et la connaissance des dispositifs numériques.

\section{La multiplicité des écrans}

La multiplicité des écrans est perçue au cours de l'évolution des technologies sous l'angle de la nouveauté, des performances et de la force de changement (Lancien, 2008). L'écran dynamique de la télévision et ses variantes numériques ou "virtuelles" (Lev Manovich, 2001) sont avant tout des objets connectés tels que les ordinateurs, les tablettes, les smart phones... L'ordinateur permet de démultiplier l'image et cette dernière peut devenir des données (textes, graphiques...). Lancien (2008), souligne que les écrans peuvent donner lieu à un autre type de catégorisation pour mieux cerner les postures spectatorielles. Il distingue :

- L'écran de représentation : principalement celui du cinéma s'inscrit dans la logique des transformations successives du travail de re-présentation comme au fait que la re-présentation suppose quelque chose d'absent ;

- L'écran de transmission : serait celui de la télévision. Il présente les images qui du point de vue de leur réception auraient tendance à s'hybrider ;

- L'écran d'action : est celui de l'ordinateur qui nécessite l'intervention du spectateur pour choisir, afficher, interrompre, fermer des documents. L'expérience spectatorielle est fortement modifiée ;

- L'écran de contact : c'est celui des téléphones mobiles. Il y serait tantôt un lieu fonctionnel (consultations, services, commandes), tantôt un lieu d'attention plus qui aurait surtout une valeur de contact.

En plus de cette description, Lancien (2008), ajoute que la multiplicité des écrans affecte les images qui y circulent ; il les distingue en images nomades, images hybrides et images nouvelles. Les images nomades 
réfèrent à des images identiques que l'on peut voir sur différents écrans ; quant aux images hybrides, elles sont issues d'un dispositif de filmage particulier lié à un certain type d'écran et viennent hybrider un autre dispositif. Enfin, les images nouvelles seraient liées principalement à l'ordinateur dont les écrans pénètrent dans l'univers de l'action. Avec les mutations imposées par le numérique, le dialogue entre l'écran et le spectateur marque le régime de la commutation qui prend la place du régime habituel de la communication.

Comme on le constate, la multiplicité des écrans affecte le rapport aux images et les postures spectatorielles. Ces rapports peuvent concerner les relations culturelles aux écrans, l'expérience cinématographique, la fréquentation télévisuelle, l'activité aux réseaux et la relation mobile selon la classification de Lancien (2008). Les différents dispositifs d'écran semblent bien d'une part s'inscrire dans différents types de relations et d'autre part affecter les images qu'ils proposent. Ce constat est également fait par les répondants de l'échantillon enquêté.

Tableau 1 : Perception des répondants de la multiplicité des écrans

\begin{tabular}{|c|c|c|c|c|}
\hline Dispositif et écran & Foyers & Etudiants & $\begin{array}{c}\text { Gestionnaires de } \\
\text { télécentres }\end{array}$ & Perception \\
\hline Ordinateur & $10 \%$ & $20 \%$ & $30 \%$ & $\begin{array}{c}\text { C'est adapté pour voir les } \\
\text { films et les documentaires }\end{array}$ \\
\hline Tablette & $10 \%$ & $20 \%$ & $30 \%$ & $\begin{array}{c}\text { Super, convient en toutes } \\
\text { circonstances }\end{array}$ \\
\hline Smartphone & $20 \%$ & $50 \%$ & $30 \%$ & $\begin{array}{c}\text { Le meilleur pour tout faire, } \\
\text { informations, films, } \\
\text { messagerie }\end{array}$ \\
\hline $\begin{array}{c}\text { Petit écran } \\
\text { (télévision) }\end{array}$ & $50 \%$ & $20 \%$ & $10 \%$ & $\begin{array}{c}\text { A la maison, en famille, } \\
\text { c'est mieux pour } \\
\text { rassembler tout le monde }\end{array}$ \\
\hline
\end{tabular}

Source : notre enquête.

Les écrans connectés sont diversement appréciés ou perçus par les répondants pour leur utilité ou leur convivialité. Ce sont les outils d'actualité pour visionner les contenus télévisuels. Seuls les étudiants et les gestionnaires des télécentres recourent aux écrans virtuels.

\section{La mise en écrans}

Les contenus télévisuels sont accessibles en mobilité sur les réseaux de téléphonie mobile. De plus les écrans de visionnage se diversifient et se nomadisent (ordinateur, baladeur vidéo, téléphone, tablette), favorisant des consommations en mobilité. Les chaînes doivent donc rénover les modalités de leur publication mais aussi leurs moyens de capter et de valoriser l'attention des utilisateurs. Trois modalités inédites de publication et leurs implications ont été mises au point : l'application enrichie, l'agrégation communicationnelle et la social TV. 
l'application enrichie

Kessous (2011) mentionne des programmes en rattrapage, des extraits et des bonus associés à certaines émissions. Nous notons également le développement des outils améliorant le confort de la navigation et du visionnage, comme la gestion des favoris, les alertes personnalisées, le contrôle du direct, le moteur de recherche interne ou encore la géolocalisation et le contrôle parental. Enfin, dans l'ensemble des applications il y a l'intégration des fonctions de partage associées à Facebook, Twitter mais aussi aux mails et aux SMS (Virginie Sonet, 2015).

\section{- L'agrégation communicationnelle}

La consultation des réseaux socionumériques (RSN) depuis les smartphones ne cesse d'augmenter ; les RSN sont aussi devenus des espaces de consommation de contenus audiovisuels. Captant ces pratiques des usagers, les chaînes de télévision ont décidé de faire de leurs contenus l'objet de ces pratiques communicationnelles, fortement prescriptrices de consommation audiovisuelle. Ces modalités d'agrégation reposent sur la logique de production de l'attention (l'alerte) inédite pour les chaînes. Dans ce régime, le contenu est adressé par un membre du réseau social de l'utilisateur. Cette personne agit alors comme un réducteur d'incertitude pour le programme : en le relayant personnellement, elle lui transfère sa propre réputation. Les récepteurs peuvent ainsi être plus enclins à visionner le programme. (Virginie Sonet, 2015).

\section{- L'écran compagnon}

La Social TV est une forme alternative de présence sur le smartphone mobilisé comme un écran annexe qui accompagne le visionnage du programme sur le téléviseur. Les chaînes conçoivent le smartphone comme un «compagnon» susceptible de diriger de nouveau l'attention de l'utilisateur sur le téléviseur. Le caractère interactif du smartphone est valorisé et les usages qui lui sont rattachés exploités. Nous avons interrogé les répondants pour connaître ce qu'ils savent des processus de mise en écrans.

Tableau 2 : Opinions des répondants sur les techniques de mise en écrans

\begin{tabular}{|c|c|}
\hline Répondants & Extraits d'entretien \\
\hline $\begin{array}{c}\mathrm{Rx} \\
\text { (non étudiants) }\end{array}$ & $\begin{array}{c}\text { Je ne sais pas comment ça fonctionne sur les cellulaire pour voir les } \\
\text { films et les autres images... mon cellulaire fonctionne bien, il n'est pas } \\
\text { encore en panne. Je ne comprends pas les choses des cellulaires }\end{array}$ \\
\hline $\begin{array}{c}\text { Ry } \\
\text { (étudiants) }\end{array}$ & $\begin{array}{c}\text { Je vois que les vidéos marchent bien sur les cellulaires. J'ai pris des } \\
\text { films avec Bluetooth sur la machine du voisin... le reste, ça, je ne } \\
\text { comprends pas. }\end{array}$ \\
\hline $\begin{array}{c}\text { Rz } \\
\text { (gestionnaires) }\end{array}$ & Les techniques utilisées pour intégrer les vidéos ou les films, je n'en \\
sais rien... Ce n'est pas mon fort.
\end{tabular}

Source : notre enquête. 
Les répondants ignorent les processus de mise en écrans. Ce n'est pas leur métier. Même les gestionnaires des télécentres ne sont pas mieux outillés que les étudiants.

\section{Les dispositifs sociotechniques}

La littérature spécialisée indique qu'une nouvelle donne médiatique est à l’origine du processus engagé par les chaînes pour élargir leur palette de contenus, intégrer de nouveaux dispositifs (télévision connectée, télévision augmentée) et diversifier leurs modes de diffusion. Des sites de téléchargement et de partage de vidéos (YouTube, Dailymotion, etc.) sont aujourd'hui (en particulier auprès des jeunes) les principaux pourvoyeurs de contenus télévisés (extraits ou originaux). Les dispositifs sociotechniques sont utilisés dans notre milieu d'étude. Nous avons donc cherché à connaître les activités des usagers. Le tableau ci-après en donne une idée.

Tableau 3 : Activités des répondants sur les dispositifs socio-techniques

\begin{tabular}{|c|c|c|c|c|}
\hline Dispositifs & Foyers & Etudiants & $\begin{array}{l}\text { Gestionnaires } \\
\text { de télécentres }\end{array}$ & Activités \\
\hline $\begin{array}{l}\text { Réseaux socio- } \\
\text { numériques }\end{array}$ & $20 \%$ & $80 \%$ & $60 \%$ & $\begin{array}{c}\text { Communiquer, } \\
\text { échanger les vidéos }\end{array}$ \\
\hline Blogs & $20 \%$ & $80 \%$ & $60 \%$ & $\begin{array}{c}\begin{array}{c}\text { Voir les informations } \\
\text { sur les films }\end{array} \\
\end{array}$ \\
\hline Page web & $0 \%$ & $80 \%$ & $60 \%$ & $\begin{array}{l}\text { Suivre les actualités sur } \\
\text { les séries }\end{array}$ \\
\hline Site web & $0 \%$ & $80 \%$ & $60 \%$ & Télécharger les films... \\
\hline Forum & $0 \%$ & $80 \%$ & $40 \%$ & $\begin{array}{c}\text { Poser des questions sur } \\
\text { les saisons }\end{array}$ \\
\hline $\begin{array}{c}\text { Video à la } \\
\text { demande (VOD) }\end{array}$ & $40 \%$ & $80 \%$ & $80 \%$ & Télécharger les vidéos \\
\hline TV sur Bouquets & $80 \%$ & $40 \%$ & $40 \%$ & Zapper \\
\hline TVR & $0 \%$ & $40 \%$ & $40 \%$ & $\begin{array}{c}\text { Visionner les vidéos le } \\
\text { week-end }\end{array}$ \\
\hline Social TV & $0 \%$ & $40 \%$ & $40 \%$ & Echanger sur le site \\
\hline CTV & $0 \%$ & $40 \%$ & $40 \%$ & $\begin{array}{c}\text { Voir les séries et les } \\
\text { saisons }\end{array}$ \\
\hline
\end{tabular}

Source : notre enquête.

Selon leur niveau de technicité ou le degré d'appropriation des outils numériques, les activités varient. Les dispositifs sociotechniques sont plus utilisés par les gestionnaires des télécentres et les étudiants que par les foyers. Ces derniers sont restés fidèles aux usages du petit écran même s'ils zappent de temps à autres avec les bouquets.

\section{La diversification des pratiques de consommation}

Nous retenons comme axes pour cette section, les pratiques individuelles et les pratiques collectives. 


\section{Les pratiques individuelles}

Les technologies numériques offrent de nouveaux écrans ordinateurs portables, téléphones mobiles, tablettes - sur lesquels le spectateur consomme des programmes télévisés de manière plus individuelle. Elles permettent également le visionnage de contenus télévisuels « à la carte ". Cette nouvelle forme de consommation des contenus a un effet sur les sociabilités. C'est un moyen d'autonomisation qui renforce la « culture de chambre ». Les jeunes, surtout, s'approprient de plus en plus les dispositifs de télévision à la demande. Ils construisent ainsi une programmation à la carte et dans un espace plus personnel. Qu'en est-il de l'échantillon à l'étude dans notre recherche ? Les résultats issus des données collectées ont permis d'élaborer le tableau suivant.

Tableau 4 : Pratiques individuelles de consommation des contenus

\begin{tabular}{|c|c|c|c|}
\hline Pratiques & Foyers & Etudiants & Gestionnaires \\
\hline Suivre les informations & $80 \%$ & $40 \%$ & $60 \%$ \\
\hline Suivre les films & $40 \%$ & $80 \%$ & $60 \%$ \\
\hline Regarder les documentaires & $20 \%$ & $80 \%$ & $60 \%$ \\
\hline Echanger les films & $0 \%$ & $80 \%$ & $60 \%$ \\
\hline Lire la messagerie & $0 \%$ & $60 \%$ & $60 \%$ \\
\hline Jeux & $0 \%$ & $60 \%$ & $60 \%$ \\
\hline Télécharger les films & $0 \%$ & $80 \%$ & $60 \%$ \\
\hline
\end{tabular}

Source : notre enquête.

Les étudiants et les gestionnaires de télécentres sont plus actifs sur les dispositifs sociotechniques que les foyers. Les activités relevant de l'usage du numérique sont plus fréquentes en milieu universitaire et dans les télécentres. Les étudiants estiment que leurs pratiques sur les objets connectés sont plus aisées du fait de l'appropriation de ces outils. Etudiants et gestionnaires, selon leurs dires, éprouvent un sentiment d'efficacité personnelle (SEP) dans l'utilisation de ces outils.

\section{Les pratiques collectives}

Nous avons retenu les pratiques des fans de séries. Les fans ont des activités créatives et collaboratives qui constituent une part importante de leurs pratiques de réception. Les fans se rassemblent en "communautés de pratiques" afin de partager leurs créations. Mélanie Bourdaa (2011), propose un regroupement des activités de fans en quatre catégories qui peuvent être complémentaires :

Les activités de création : les fans mettent en place des sites Internet consacrés à leurs séries ou à leurs personnages préférés, qu'ils alimentent régulièrement en articles d'analyse ou anecdotes. Outre la création de fan vidéos ou de fan arts, ils écrivent également des fan fictions. La fan fiction est une activité d'écriture, et de réinterprétation des fans. 
Les créations de lien social : les réseaux sociaux permettent aux fans de prolonger leur expérience télévisuelle et de recréer une expérience de lien social. En recréant une "télévision cérémonielle", les fans se retrouvent autour d'un objet commun et dans un lieu commun pour débattre des épisodes qu'ils sont en train de regarder, rejouant les conditions d'un visionnage commun en direct.

Les créations de partage : l'activité principale des fans est de mettre les épisodes de série les plus récents à disposition des autres fans de la communauté. Pour développer une réception transnationale et qui ne dépend pas des contraintes de diffusion locale, les fans mettent les épisodes en ligne et des équipes de fansubbers se chargent de proposer les sous-titres, indispensables à la bonne compréhension de la série. Ces équipes de fansubbers possèdent une hiérarchie établie et chaque personne a des compétences spécifiques : traducteur, relecteur, encodeur.

Les créations d'"intelligence collective" : face aux développements des extensions transmédiatiques et des expériences Deep Media, les fans se constituent en force collaborative pour résoudre les puzzles des univers sériels proposés. Pour mutualiser leurs efforts et mettre en commun leurs découvertes, ils créent des wikis. Les fans se répartissent également en groupe pour avancer dans leurs recherches : on trouve les organisateurs qui modèrent la communauté, les détectives qui tentent de résoudre les énigmes, les chasseurs qui récupèrent les données éparpillées sur les plateformes médiatiques, et les "voyeurs" (lurkers) qui regardent les progrès de la communauté sans participer.

On retiendra que les fans sont des téléspectateurs particuliers ; non seulement ils sont experts d'un programme, mais en plus ils sont actifs dans leur réception. Cependant, ce qui les distingue des autres téléspectateurs, c'est qu'ils possèdent les compétences techniques et technologiques pour participer, interagir et se créer un environnement télévisuel personnalisé.

En utilisation individuelle ou collective, l'appropriation accrue résulte du sentiment d'efficacité personnelle (SEP) éprouvé par les usagers. Ce concept peut se définir comme la perception qu'un individu entretient quant à sa capacité à utiliser un ordinateur (Compeau, Higgins \& Huff, 1999, cités par Faurie, 2007). Outre les usages, le SEP influencerait également le niveau d'anxiété. Un SEP élevé dans l'utilisation de l'informatique entraînerait une plus grande intensité d'usage ainsi qu'une moindre anxiété face aux ordinateurs, moindre anxiété qui encouragerait elle aussi l'engagement du sujet dans l'usage du système informatique (voir figure ci-après).

Modèle du sentiment d'efficacité personnelle face aux technologies 


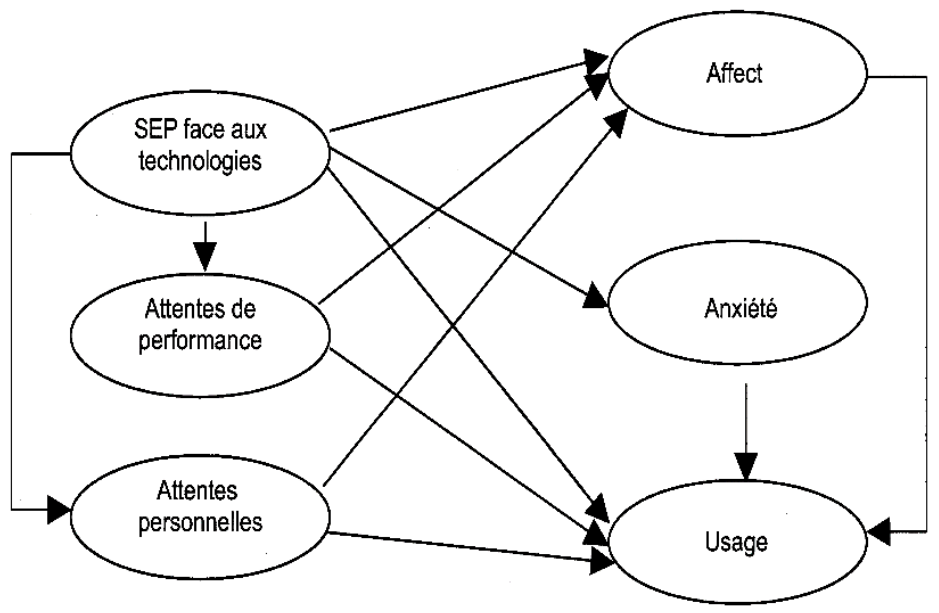

Source : inspiré de Isabelle Faurie, 2007.

Les attentes d'un individu vis-à-vis d'un comportement dépendraient des perceptions qu'il a quant à ses capacités à réaliser avec succès ce comportement. Les attentes personnelles font référence aux croyances de l'individu quant aux conséquences que l'usage de la technologie est susceptible d'entraîner (diminution ou renforcement du soutien social) et sur sa satisfaction personnelle liée au grand sentiment d'accomplissement. Notre recherche a eu recours à ce modèle en rapport avec les attentes personnelles et les attentes psychosociales ; tant cette dimension paraît importante dans le cadre des usages des dispositifs socio-techniques. La variabilité des niveaux de SEP dans les pratiques de réception des spectateurs entraîne des "postures spectatorielles" diverses (Perticoz et Dessinges, 2015) :

- $\quad$ la "téléspectature" (autonomie) : est un plan dans lequel s'inscrit le téléspecteur pour visionner ses contenus sur une multitude d'écrans ;

- _ la "téléaction" (interfaces connectés) : est un plan dans lequel s'inscrit le téléacteur pour visionner ses séries sur des écrans multiples et connectés ; - _ la "télécréation" (participation) : est un plan dans lequel s'inscrit le télécréateur pour visionner ses séries sur des écrans multiples et connectés.

Les auteurs précisent bien que leur ambition est de procéder à une clarification terminologique afin d'appréhender l'évolution des modes de consommation de fictions audio visuelles.

\section{Conclusion}

Les mutations en cours de l'objet télévisuel concernent les nouveaux dispositifs, les contenus télévisuels, les stratégies des chaînes et les accès massifs au web. En outre, les usages des dispositifs, les modes de réception et de visionnement (participation, partage, création...) se dessinent continuellement. Les processus de diffusion exploitant les écrans multiples, 
la mise en écran et les dispositifs socio-techniques sont influencés par la convergence médiatique en ses deux composantes que sont la logique technologique et la logique culturelle. Par ailleurs, la diversification des pratiques de consommation se décline en pratiques individuelles et pratiques collectives en lien avec les postures spectatorielles. Ces postures résultent de la mise en œuvre des compétences techniques des usagers sous-tendues par le sentiment d'efficacité personnelle face aux technologies.

La stratégie de recherche utilisée pour obtenir ces résultats a combiné les techniques documentaires, l'observation de terrain, l'administration de questionnaires et les entretiens. Mais, le rythme de changement des technologies numériques impose un réajustement continuel dans la méthodologie utilisée pour investiguer les processus de production des contenus télévisuels et les pratiques de visionnement. La mise en place d'un dispositif de recherche dans lequel plusieurs cas sont étudiés avec leurs dimensions contextuelles et circonstancielles (Giroux, 2003) s'avère donc indispensable pour comprendre le phénomène à l'étude dans toute sa complexité. Perticoz et Dessinges (2015) s'inscrivent dans cette logique quand ils disent : " car si les mutations de la filière doivent être entendus comme une conséquence de l'évolution des modes de consommation sérielles rendus possibles grâce à Internet et au numérique, il convient alors d'élaborer des outils méthodologiques permettant de les appréhender dans toute leur finesse et dans toute leur complexité. ». Des pistes de recherche futures sont donc ouvertes et elles resteront fortement dépendantes de l'évolution du numérique.

\section{References:}

1. Amandine Kervella et Marlène Loicq, (2015), " Les pratiques télévisuelles des jeunes à l'ère du numérique : entre mutations et permanences », Études de communication, n44, pp.79-96.

2. CNC, (2012), Les nouveaux usages de la télévision connectée, http://www.csa.fr/Espace-Presse/Conferences-de-presse/Presentationdes-travaux-de-la-Commissionde-suivi-des-usages-de-la-televisionconnectee, consulté le 29 novembre 2015.

3. Elizabeth Bastos Duarte et al., (2007), « Sur la convergence médiatique ", Télévision $n^{\circ}$ 2, p. 129-133.

4. Elodie Kredens et Florence Rio, (2015), " La télévision à l'ère numérique : entre pratiques émergentes et reconfiguration de l'objet médiatique », Études de communication, n44, pp. 15-28.

5. François Jost, (2015), "Quelle relation au temps nous promet-on à l'ère de l’ubiquité télévisuelle ? », Télévision nº 6, p. 101-113. 
6. Guillaume Blanc, (2015), « Les pratiques de réception télévisuelle dans les foyers à l'épreuve de l'audiovisuel numérique », Études de communication

7. Henry Jenkins, (2013), La Convergence culturelle, Nouveaux Médias, nouveaux publics, Armand Colin.

8. Isabelle Faurie et Cécile van de Leemput, (2010), "Influence du sentiment d'efficacité informatique sur les usages d'internet des étudiants », L'orientation scolaire et professionnelle, 36/4 | 2007.

9. Jost F., (2014), La télévision et après : vers le transmedia, Télévision, $\mathrm{n}^{\circ}$ 5, CNRS Editions, $162 \mathrm{p}$.

10. Lancien Th., (2011), Multiplication des écrans, images et postures spectatorielles, Conférence faite au colloque de l'Afeccav : «Faire de la recherche en cinéma et en audiovisuel : quelles pratiques pour quels enjeux ?», 9-10-11 juillet 2008. http://intermedialite.blogspot.fr/2009/10/multiplication-des-ecransimages-et.html, consulté le 2 décembre 2015.

11. Lucien Perticoz et Catherine Dessinges, (2015), « Du téléspectateur au télé-visionneur. Les séries télévisées face aux mutations des consommations audiovisuelles ", Études de communication, $\mathrm{n}^{\circ} 44$, pp. 115-130.

12. Bourdaa Mélanie, (2011), « Les nouvelles pratiques de production et de réception télévisuelles », http://www.inaglobal.fr/television/article/les-nouvelles-pratiques-deproduction-et-de-reception-televisuelles?tq=1, consulté le 15 mars 2016.

13. Myriam Montagut-Lobjoit, (2010), « La télévision au prisme des TIC. Pratiques et perspectives ", Les Cahiers du numérique, (Vol. 6), p. $99-121$.

14. Virginie Sonet, (2015), « La télévision sur smartphone : ressorts et implications du renouvellement des modalités d'agrégation », Études de communication, ${ }^{\circ} 44$, pp. 47-62. 